\title{
The Movement ABC-2 Test in China: comparison with UK norms for 3-10 year olds
}

Li Keㄹ, Wenchong Du ${ }^{2 *}$, Yun Wang ${ }^{3}$, Wen Duan ${ }^{1}$, Jing Hua ${ }^{4}$, Anna L. Barnett ${ }^{5}$

1 Collaborative Innovation Center of Assessment for Basic Education Quality, Beijing Normal University, China

2 Department of Psychology, Nottingham Trent University, UK

3 State Key Laboratory of Cognitive Neuroscience and Learning, Beijng Normal University, China

4 Shanghai First Maternity and Infant Hospital, Tongji University School of Medicine, China

5 Department of Psychology, Health and Professional Development, Oxford Brookes University, UK

*Corresponding author, Department of Psychology, Nottingham Trent University, Burton Street, Nottingham, NG1 1BU, UK, e-mail: vivienne.du@ntu.ac.uk

Declarations of interest: none.

\section{Abstract}

The Test component of the Movement Assessment Battery for Children $2^{\text {nd }}$ Edition (Movement ABC-2) is used worldwide to identify children with Developmental Coordination Disorder (DCD). In China, practitioners have been using this test with the assumption that the published UK norms are valid for Chinese children. However no systematic investigation has previously been undertaken to check this assumption. 2185 children aged 3-10 years old from a national representative sample in China were therefore recruited to the current study. Performance on the Movement ABC-2 was assessed and compared with the UK standardization norms. Gender differences were 
also examined. The comparisons revealed that Chinese children were generally better in Manual Dexterity and Balance tasks compared to their UK peers; while UK children were better in Aiming \& Catching tasks. Further analysis showed an interaction of country and age with mixed results. For both countries, girls were generally better in Manual Dexterity and Balance tasks, and boys were generally better in Aiming \& Catching. Possible explanations for the country differences are discussed. The results suggest that local norms for the Movement ABC-2 Test are needed in China.

\section{What this paper adds?}

Previous studies have reported cross-cultural differences between Western and East Asian children when assessing their motor performance with the first and second editions of the Movement ABC Test. However the results were mixed in terms of different age groups and different motor components. Children with Developmental Coordination Disorder (DCD) have recently started to be recognised in the healthcare and education system in mainland China, and the Movement ABC Test has been widely used by researchers and practitioners. However, little is known about the suitability of applying the published UK norms of the assessment on the Chinese population. Using a national representative sample, the current study provided original data on the cultural differences in motor development between Chinese children and their UK peers on the Movement ABC-2 Test. We propose that the more intensive training program in nurseries in China might partially explain some of the differences found between children from the two countries.

\section{Highlights:}

- Chinese \& UK children aged 3-10 have different profiles on the Movement ABC-2 Test;

- Gender differences in motor development are consistent across China and the UK;

- Local norms for the Movement ABC-2 Test are needed in China. 


\section{Introduction}

The Movement Assessment Battery for Children (Movement ABC) Test and the revised second edition (Movement ABC-2) ${ }^{1}$ (Henderson \& Sugden, 1992; Henderson, Sugden, \& Barnett, 2007) have been used worldwide in the identification and assessment of children with movement and coordination impairments. The Movement ABC-2, designed for use with 3 to 16-year-old children, has eight test items in each of three age bands (for 3-6, 7-10, and 11-16 years). Within each age band, the three-part structure of the Movement ABC-2 is theoretically identical, with Manual Dexterity (MD), Aiming and Catching (AC), and Balance (BAL) components. The Movement ABC-2 has been one of the tests highlighted in international recommendations for the assessment of Developmental Coordination Disorder (DCD), a common condition in childhood impacting on everyday motor performance (Blank, Smits-Engelsman, Polatajko, \& Wilson, 2012; Blank, et al., 2019).

The Movement $\mathrm{ABC}$ and Movement ABC-2 Tests were published with US and UK norms respectively. Various studies have suggested that the norms may need to be adjusted when used in different cultures (Ruiz, Graupera, Gutiérrez, \& Miyahara, 2003). Each culture has its own distinctive pattern of teaching practices and encourages different behaviors and skills in children. In the domain of motor development, differences in children's physical development may also affect the development of motor skills (Paciorek, Stevens, Finucane, Ezzati, \&Nutrition Impact Model Study Group, 2013), and different activities that children engage in within different cultures are also likely to influence children's motor development (Yan \& McCullagh, 2004). Therefore, children from different cultures may have varying motor development profiles and it may therefore not be appropriate to use the same test norms for all populations.

Previous studies have reported the cross-cultural differences between Western and East Asian children when assessing their motor performance with the Movement ABC Test (Miyahara, et al., 1998; Ruiz, Graupera, Gutiérrez, \& Miyahara, 2003; Chow, Henderson, \& Barnett, 2001; Chow, Hsu, Henderson, Barnett, \& Lo, 2006). For example,

\footnotetext{
${ }^{1}$ The first edition of the test, Movement ABC was published in 1992 with US norms; the second edition, Movement ABC-2 was published in 2007 with UK norms.
} 
children from Japan aged 7-8 years old were compared with their US peers with the first edition of Movement ABC (Miyahara, et al., 1998), and the results showed that Japanese children tended to perform better in Dynamic Balance tasks, while US children tended to do better in Manual Dexterity tasks. Also with the first edition of Movement ABC, Chow et al. (2001) reported that 255 children from Hongkong performed significantly better than their US counterparts $(n=799)$ on items related to Manual Dexterity and Dynamic Balance; while the US children performed better on items relating to Aiming and Catching. Moreover, an interaction between country and age were also found with some tasks, which suggests a difference in children's movement development from different cultures. In a later report, Chow et al. (2006) added an extra sample from Taiwan (544 children) and compared performance on the Movement ABC Test with the US sample. The results were similar to their earlier report with children from Hong Kong only. Interestingly, a significant difference was found between the performance of children from Hong Kong and Taiwan on some tasks, which suggests that although sharing a common language and belonging to the same ethnic group, cross-cultural differences may still exist.

More recent studies using the Movement ABC-2 with Japanese children reported similar results in that Japanese children aged 7-11 years old were found to perform better in component scores using the UK norms with respect to Manual Dexterity and Balance (Kita, et al., 2016). With regard to gender differences, in both Japanese and UK groups, girls obtained superior Manual Dexterity and Balance scores compared with boys. In a later study also with the Movement ABC-2, 252 Japanese children aged 3-6 years old were found to have higher Manual Dexterity and Balance scores than children from the UK normative sample. Girls scored better than boys on the Balance components (Hirata, et al., 2018). With these studies, it seems that East Asian children tend to have better performance in Manual Dexterity and Balance components compared to US or UK children; and a consistent cross-cultural gender difference also exists. The universal gender differences in motor development across cultures used to lead to an argument that separate norms for the Movement $\mathrm{ABC}$ and other motor assessments should be developed for boys and girls (Dunn \& Watkinson, 1996). However, the main purpose for the Movement $A B C$ is to identify movement and coordination difficulties (Henderson \& Sugden, 1992; Henderson, Sugden, \& Barnett, 2007), and it may not be appropriate to have separate norms for girls and boys 
especially when an estimate of the incidence of motor impairments by gender is unclear (Cairney, Hay, Faught, Mandigo, \& Flouris, 2005).

Over the past decade in mainland China, children with DCD have started to be recognized in the healthcare and education system, and the Movement ABC-2 Test has been widely used by researchers and practitioners. Simultaneously, researchers have started to look at the suitability of the Movement ABC-2 Test for Chinese children. In 2013, Hua, Gu, Meng, and Wu collected data on the Movement ABC-2 Test from 1956 children aged 3-6 years old in southern China, and reported good reliability and validity of the test (Hua, Gu, Meng, \& Wu, 2013). In a later study, Hua et al. (2014) collected data from 4416 children aged 3-6 years old in Suzhou City in China, and found that the onechild family status was significantly related to children's motor development and DCD. Therefore they suggested China might generally have less developed movement skills because the majority of Chinese children are the only child in their family which might negatively affect their movement development. However, a direct comparison of children from China and other countries is needed to support this argument. Moreover, no data on motor development has yet been reported separately for males and females in the Chinese population, so it is not known whether or not Chinese children show similar gender differences as western children.

Hua et al's studies have confirmed the good validity and reliability of the Movement ABC-2 Test for Chinese children. Other previous studies on East Asian populations have suggested significant differences on component scores compared to western norms. The aims of the current study were therefore to (i) select a national representative sample of children aged 3 to10 years in China, who were assessed on the Movement ABC-2 Test, (ii) compare data with the UK standardization norms; and (iii) examine gender differences.

\section{Method}

\subsection{Participants}

Data from 765 children between the age of 3 and 10 years (i.e., Age Band 1 and 2) were included from the UK standardization sample of the Movement ABC-2 Test (387 boys, 378 girls). A stratified sampling plan was used to ensure the Chinese sample for 
the current study was representative of the Chinese population. Similar stratification variables were used as for the UK standardisation. Data from the 2010 National Census in China provided the basis for the stratification by geographic region, age, gender, and social economic status (SES). Since China is considered as a mono-ethnic country (Central Intelligence Agency, 2018), ethnicity was not included as a stratification variable. The target population was children aged 3:0-10:11 years (Age Bands 1 and 2) from urban areas of China.

Within the overall stratified sampling structure, children were sought whose demographic characteristics best fitted the requirements in terms of gender and age from 3:0-10:11 years inclusive. Following the structure of UK sampling, at age 3 and 4, each age group was divided into two so that norms could be provided for half-year groups. Thereafter, there were a total of 10 levels of age. According to the 2010 Census, the proportion of the Chinese population between boys and girls are the same for all 10 age levels. Table 1 shows the breakdown of number of children recruited in each age and gender group. More information of the participants' geographic and SES information can be found in the Appendix.

(insert Table 1 here)

\subsection{Materials}

The first two age bands of the Movement ABC-2 Test were used in the current study: $A B 1(3: 0-6: 11$ years); and $A B 2$ (7:0-10:11 years). For each age band, there are eight tasks: 3 tasks measure manual dexterity (MD) (posting coins/placing pegs; threading lace; drawing); 2 tasks measure ball skills (BS) (throwing/aiming and catching); and 3 tasks measure balance (B) (one or two leg balance; walking along a line; jumping or hopping). For each task, a raw score is obtained. This varies across the tasks and includes overall speed (time in seconds), accuracy (number of errors) or the number of correctly completed movements. The raw scores obtained for each task are summarized in Table 2 below.

(insert Table 2 here) 


\subsection{Recruitment procedure, quality control, and administration}

Nurseries and schools were sampled to include children of all ages, and invitations to participate in the project were made by email and telephone to nursery and primary schools chosen to be representative of our targeted sample. Consent forms and instructions for distributing these for whole classes of children were delivered to participating nurseries and schools. Consent was obtained from both nurseries/schools and parents. Verbal assent was also provided by each participant.

Permission was obtained from the test publisher to translate the record forms and the test instructions from English into Chinese; common use of terms (e.g., the name and description of tasks in Chinese translation ) from earlier publications in Chinese was used in the translation. A back-translation from Chinese to English was undertaken by independent translators, and the original and back-translated versions were equivalent in meaning. An expert panel with experts in relevant fields was convened to review every step towards the Chinese translation of Movement ABC-2. All 35 assessors had proficient experience in conducting psychological assessments with children in a similar age range, and all assessors were trained to individually administer the Movement ABC-2 Test with a two-day training program.

In all cases, children were assessed individually in their own nurseries/school. The testing duration for each child was 30-40 minutes. Ethical approval was obtained by the Institutional Review Board (IRB) of the National Key Laboratory of Cognitive Neuroscience and learning, Beijing Normal University.

\section{4. $\quad$ Scoring}

The scoring was consistent with the method published in the Movement ABC-2 UK manual. Failed/refused trials were recorded in the same way as in the published UK norms: 1) if a "fail" was recorded for a trial, the lowest score was given for that trial; 2) if a "refuse" was recorded for a trial, if the child was obviously doing badly on other tasks, the lowest possible score on the refused item was assigned; if the child was doing quite well on other tasks, the mean for the group as an estimate of the performance was 
assigned to that refused trial. There are 8 tasks in the Movement ABC-2 Test, however, for AB1, there are two scores recorded for two tasks (Posting Coins, One-leg Balance) with preferred/nonpreferred or best/the other hand/leg, therefore 10 scores were considered as dependent variables for AB1. For AB2, two scores are recorded for three tasks (Placing Pegs, One-board Balancing, Hopping on Mats), therefore 11 scores were considered as dependent variables for AB2. In all analyses, each of the 21 measures have been considered separately.

\subsection{Data analysis}

A MANOVA was performed on all of the 10 scores to test the effects of Age, Country, and Gender. Bonferroni tests were conducted for all further post hoc checks, $p<.05$ was considered as significant. All analyses were performed using IBM SPSS Statistics 25.

\section{Results}

Table 3 and Table 4 presents the means and standard deviations of the children's raw scores on each task in $\mathrm{AB} 1$ and $\mathrm{AB} 2$.

(insert Table 3 here)

(insert Table 4 here)

For AB1, a main effect of age was found on all tasks, with older children performing better than younger children. As shown in Table 5, a main effect of Country was found on several tasks: Chinese children showed better performance on Posting Coins (for both preferred and non-preferred hand), One-leg Balance, and Jumping on Mats, while UK children had a better performance on Drawing. A main effect of Gender was also found on several tasks: girls generally performed better than boys on Threading, Drawing, One-leg Balance, Walking Heels Raised, and Jumping on Mats, while boys had better performance on Throwing Beanbags.

Significant interactions were found with some tasks (Table 6), simple main effects showed that for Manual Dexterity, and Posting Coins with preferred hand, Chinese children performed better at 3.5, 4, 4.5, 5, and 6 years compared to UK children; while for Drawing, Chinese children performed worse than UK children at 3, 5, and 6 years. In 
Aiming \& Catching, for Throwing Beanbag, UK children performed better than Chinese children at 3, 3.5, and 4 years, while Chinese children performed better than UK children at 5 and 6 years. For Balance, both One-leg Balance tasks showed the same trend: Chinese children performed worse than UK children at 3 years but better than UK children at 3.5, 4, 5, and 6 years. For Jumping on Mats, girls performed better than boys at 3 and 3.5 years but no difference was found at other ages.

(insert Table 5 here)

(insert Table 6 here)

For AB2, for all tasks, there was a significant effect of age, with older children performing better than younger children. There was a significant main effect of Country on some tasks: Chinese children performed better than UK children in Placing Pegs, One-board Balance-best leg, and Hopping on Mats; while UK children performed better than Chinese children on both Aiming \& Catching measures (Catching and Throwing Beanbag). There was a significant effect of Gender on some tasks: girls were better than boys on preferred-hand Placing Pegs, Threading Lace, One-board Balancing, Walking Heels-to-Toe Forwards, and Hopping on Mats; while boys were better than girls on both Aiming \& Catching measures (Catching, and Throwing Beanbag) (Table 7).

Significant interactions were found for some tasks (Table 8); simple main effect analyses show that for Manual Dexterity, Chinese children performed better than UK children only at 9 and 10 years on Placing Pegs with preferred hand, but not at 7 and 8 years. For Aiming \& Catching, girls performed worse than boys only on Catching at 9 and 10 years. For One-board Balance-best leg, girls performed better than boys at 7,8 and 9 years but no difference was found at 10 years.

(insert Table 7 here)

(insert Table 8 here)

\section{Discussion}

The aim of this study was to use a national representative sample of children aged 3 to10 years in China to compare data on the Movement ABC-2 with the UK standardization norms and to examine the effect of gender. Our results suggested a 
significantly different motor development profile between Chinese and UK children. Although there were mixed results across the different tasks in the three motor domains within the Movement ABC-2 Test, in general, Chinese children performed better in Manual Dexterity and Balance in both age bands; while only for children in the older age band (AB2), UK children performed better in Aiming \& Catching. In both age bands and both countries, in general, girls were better in Manual Dexterity and Balance tasks; while boys were better in Aiming and Catching tasks.

With regard to cultural differences between the Chinese and UK children, with a national representative sample, we found the Chinese children showed a better performance in Manual Dexterity tasks; Chinese children also performed better compared to their UK peers in both Static and Dynamic Balance tasks. The results were consistent with the recent studies on Japanese children with the Movement ABC-2 Test (Kita, et al., 2016; Hirata, et al., 2018); but different from the studies which only reported a better performance of East Asian children in Dynamic but not Static Balance tasks with the first edition of the Movement ABC (Chow, Henderson, \& Barnett, 2001; Chow, Hsu, Henderson, Barnett, \& Lo, 2006; Miyahara, et al., 1998). The reasons behind these reported differences between East Asian and western (US or UK) children are likely to be multifactorial.

Within all the studies which have been reviewed in this article, data from mainland China, Japan, Hong Kong, and Taiwan have been included. It should be noted that more consistent results were only achieved from more recent studies with the Movement ABC-2 Test (Hirata, et al., 2018; Kita, et al., 2016) compared to the earlier studies using the first edition of the Movement ABC Test. One explanation might relate to the different editions of the Movement ABC-2 Test. Although the first and second edition of the Test are very similar, there are some differences in the age bands used ${ }^{2}$ and the norms were collected from different countries (the US and the UK). Secondly, there may be a change in children's motor development over the past decades, with the motor development profile of children nowadays possibly being different compared to previous years in different cultures. Some differences between the East Asian and western samples might be due to changes over time because the education and living environment has changed dramatically in the past 20 years in some countries. Therefore, we suggest that the most

\footnotetext{
2 Movement ABC had four age bands (4-6, 7-8, 9-10, 11-12 years) while Movement ABC-2 has three age bands $(3-6,7-10,11-16$ years $)$.
} 
updated assessment should always be used, and the Movement ABC-2 Test might need to be updated with more recent norms.

Furthermore, analysis of the cross-cultural differences across the age groups revealed more details of the motor development profile of Chinese children. Within AB1, the younger (3-4-year-old) Chinese children had poorer scores compared to their UK peers in Drawing, Throwing Beanbag, and One-leg Balance; but at 5 and 6 years, Chinese children were better than their UK peers on those tasks. Conversely, in AB2, from 7 years old, Chinese and UK children started to show more similar development curves across all age groups with a better performance of Chinese children in Manual Dexterity and Balance, and a better performance of the UK children in Aiming \& Catching. In China, children usually attend nursery from age 3 to 6 years and primary school from 7. Tso et al. (2018) using the Griffiths Development Scales, compared Chinese and UK children aged 2-8 years old, and reported similar results. They found that young Chinese children showed poorer scores on motor skills compared to their UK peers at 2 years old, but the UK children showed a steadier growth rate while the Chinese children exhibited a steeper growth rate and the two sets of curves converged after 6 years of age. The explanation Tso et al. provided for the younger Chinese children's poorer initial motor performance at an early age was consistent with Hua et al.'s explanation (Hua, et al., 2014), that Chinese parents tend to "overprotect" and "spoil" their only child under the one-child policy especially at the very early stage, thus children in China had limited chances to practice gross or fine motor skills to avoid any potential injury (Tso, et al., 2018).

However, what is the reason for the steeper rate of development of Chinese children after the age of 5 years suggested in our data? In China, in the last 1-2 years in the nursery (5-6 years old), "transition to school" has become the key target and more target-oriented education programmes have started to launch from that stage (Yang, 2013). Nurseries deliver more structured and intensive training programmes to older children to prepare the children for school with developed linguistic, mathematics, and motor skills, etc. All of our participants were recruited from nurseries and followed the education structure of the "transition to school" programme; therefore, it is possibly because of the introduction of a more intensive training programme in the later years in nurseries that we see a steeper growth rate of Chinese children. After 7 years of age, both Chinese and UK children were attending primary school and the cross-cultural 
differences became more stable. The same results were reported in Japanese children (Kita, et al., 2016; Hirata, et al., 2018), with East Asian children showing generally better performance in Manual Dexterity and Balance, and worse Aiming and Catching performance compared to their UK peers.

Our study also found that girls generally performed better in Manual Dexterity and Balance; while boys were generally better in Aiming and Catching, with both countries and both age bands showing the same results. Again, our results were consistent with the previous two studies on Japanese children using the Movement ABC-2 (Kita, et al., 2016; Hirata, et al., 2018). Similar results have also been reported that girls tend to be better than boys on Manual Dexterity and Balance but not those tasks involving throwing and catching (Chow, Henderson, \& Barnett, 2001; Chow, Hsu, Henderson, Barnett, \& Lo, 2006; Miyahara, et al., 1998; Junaid \& Fellowes, 2006; Engel-Yeger, Rosenblum, \& Josman, 2010). It is difficult to draw any conclusion regarding the gender difference, for example, some studies have claimed that gender differences in prepubescent school-aged children can be attributed more to social and environmental factors than to biological factors (Garcia, 1994). Therefore, the universal gender difference found across cultures and confirmed by the current study might suggest that gender differences in socialization are rather consistent across the different cultures which have been included in previous studies. More evidence is needed from a culture with different social gender roles in childhood.

In summary, as a preliminary examination of the suitability of the UK norms of the Movement ABC-2 Test for Chinese children, a significant difference has been found between the Chinese and the UK normative sample across different motor domains. This suggests the need to develop Chinese norms to use the Movement ABC-2 Test with the Chinese population. Our study can serve as an initial step for the standardization process and further studies will be needed to develop a Chinese version of the Movement ABC-2 Test in the future.

\section{Acknowledgments}

The authors wish to thank all researchers, teachers, and assessors who participated in this study for the collaboration and data collection. We also thank the expert panel, Prof. Yun Wang, Prof. Gui Xue, Prof. Guixiong Gu, Prof. Liang Luo, Prof. Hui Zhao, Prof. 
Ping Ren, Dr. Cai Zhang, and Dr. Hua Jin; and three authors of Movement ABC-2, Prof.

Sheila E. Henderson, Prof. David A. Sugden, and Prof. Anna L. Barnett, for their

professional consultation and support to the project. This work was supported by

Leiton Ltd. and Pearson Assessment, as part of the development of the Chinese

Movement ABC project.

\section{Author statement}

Li Ke: Validation, Investigation, Data Curation, Writing - Review \& Editing. Wenchong Du: Conceptualization, Methodology, Funding acquisition, Writing - Original Draft, Writing - Review \& Editing. Yun Wang: Resources. Wen Duan: Investigation, Formal analysis, Data Curation, Project administration. Jing Hua: Methodology, Writing Review \& Editing, Anna Barnett: Writing - Review \& Editing, Supervision.

\section{References}

American Psychiatric Association. (2013). Diagnostic and statistical manual of mental disorders. American Psychiatric Pub.

Blank, R. B., Rosenblum, S., Smits-Engelsman, B., Sugden, D., Wilson, P., \& Vinçon, S. (2019). International clinical practice recommendations on the definition, diagnosis, assessment, intervention, and psychosocial aspects of developmental coordination disorder. . Developmental Medicine \& Child Neurology, 61(3), 242-285.

Blank, R., Smits-Engelsman, B. O., Polatajko, H., \& \& Wilson, P. (2012). European Academy for Childhood Disability (EACD): Recommendations on the definition, diagnosis and intervention of developmental coordination disorder (long version). Developmental Medicine \& Child Neurology, 54(1), 54-93.

Cairney, J., Hay, J., Faught, B., Mandigo, J., \& Flouris, A. (2005). Developmental coordination disorder, selfefficacy toward physical activity, and play: Does gender matter? Adapted Physical Activity Quarterly, 22(1), 67-82.

Central Intelligence Agency. $(2018,1128)$. Retrieved from The World Factbook: www.cia.gov.

Chow, S. M., Henderson, S. E., \& Barnett, A. L. (2001). The Movement Assessment Battery for Children: A comparison of 4-year-old to 6-year-old children from Hong Kong and the United States. The American Journal of Occupational Therapy, 55(1), 55-61.

Chow, S. M., Hsu, Y. W., Henderson, S. E., Barnett, A. L., \& Lo, S. K. (2006). The movement ABC: A crosscultural comparison of preschool children from Hong Kong, Taiwan, and the USA. Adapted physical activity quarterly, 23(1), 31-48.

Dunn, J. C., \& Watkinson, E. J. (1996). Problems with identification of children who are physically awkward using the TOMI. . Adapted Physical Activity Quarterly, 13(4), 347-356.

Engel-Yeger, B., Rosenblum, S., \& Josman, N. (2010). Movement Assessment Battery for Children (M-ABC): establishing construct validity for Israeli children. Research in Developmental Disabilities, 31(1), 87-96.

Engel-Yeger, B., Rosenblum, S., \& Josman., N. (2010). Movement assessment battery for children (M-ABC): Establishing construct validity for Israeli children. Research in Developmental Disabilities, 31, 8796.

Garcia, C. (1994). Gender differences in young children's interactions when learning fundamental motor skills. Research Quarterly for Exercise and Sport, 65(3), 213-225.

Geuze, R. H., Jongmans, M. J., Schoemaker, M. M., \& Smits-Engelsman, B. C. (2001). Clinical and research diagnostic criteria for developmental coordination disorder: a review and discussion. Human movement science, 20(1-2), 7-47.

Henderson, S. E., \& Sugden, D. A. (1992). Movement assessment battery for children. Kent, UK: The Psychological Corporation. 
Henderson, S., Sugden, D. A., \& Barnett, A. L. (2007). Movement assessment battery for children-2 second edition [Movement $A B C$-2]. London, UK: The Psychological Corporation. .

Hirata, S., Kita, Y., Yasunaga, M., Suzuki, K., Okumura, Y., Okuzumi, H., . . Nakai, A. (2018). Applicability of the Movement Assessment Battery for Children-(MABC-2) for Japanese children aged 3-6 years: a preliminary investigation emphasizing internal consistency and factorial validity. Frontiers in psychology, 9.

Hua, J., Gu, G., Jiang, P., Zhang, L., Z. L., \& Meng, W. (2014). The prenatal, perinatal and neonatal risk factors for children's developmental coordination disorder: a population study in mainland China. Research in developmental disabilities, 35(3), 6.

Hua, J., Gu, G., Meng, W., \& Wu, Z. (2013). Age band 1 of the Movement Assessment Battery for Children-: Exploring its usefulness in mainland China. Research in Developmental Disabilities, 34(2), 801808.

Hua, J., Jin, H., Gu, G., Liu, M., Zhang, L., \& \& Wu, Z. (2014). The influence of Chinese one-child family status on Developmental Coordination Disorder status. Research in developmental disabilities, 35(11), 3089-3095.

Junaid, K. A., \& Fellowes, S. (2006). Gender differences in the attainment of motor skills on the movement assessment battery for children. . Physical \& Occupational Therapy in Pediatrics, 26(1-2), 5-11.

Kirby, A. \&. (2007). Children with developmental coordination disorders. Journal of the royal society of medicine, 100(4), 182-186.

Kita, Y., Suzuki, K., Hirata, S., Sakihara, K., Inagaki, M., \& \& Nakai, A. (2016). Applicability of the Movement Assessment Battery for Children-to Japanese children: A study of the Age Band 2. Brain and Development, 38(8), 706-713.

Miyahara, M., Tsujii, M., Hanai, T., Jongmans, M., Barnett, A., Henderson, S. E., \& ... \& Kageyama, H. (1998). Miyahara, M.; Tsujii, M.; Hanai, T.; Jongmans, M.; Barnett, A.; Henderson, S.E.; Hori, M.; Nakanishi, K; Kageyama, H. Human Movement Science, 17(4-5), 679-697.

Paciorek, Stevens, Finucane, Ezzati, \&Nutrition Impact Model Study Group. (2013). Children's height and weight in rural and urban populations in low-income and middle-income countries: a systematic analysis of population-representative data. The Lancet Global Health, 1(5), e300-e309.

Ruiz, L. M., Graupera, J. L., Gutiérrez, M., \& Miyahara, M. (2003). The Assessment of Motor Coordination in Children with the Movement ABC test: A Comparative Study among Japan, USA and Spain. International Journal of Applied Sports Sciences, 15(1).

Tso, W. W., B., Li, M., Xu, X., Ao, L., Zhang, X., . . Shang, X. (2018). The Griffiths Development Scales Chinese (GDS-C): A cross-cultural comparison of developmental trajectories between Chinese and British children. Child: care, health and development, 44(3), 378-383.

Wilson, B. N., Kaplan, B. J., Crawford, S. G., Campbell, A., \& Dewey, D. (2002). Reliability and validity of a parent questionnaire on childhood motor skills. American Journal of Occupational Therapy. 54(5), 484-493.

World Bank. (2016). Rural population (\% of total population). Retrieved from https://data.worldbank.org/indicator/sp.rur.totl.zs

Yan, J. H., \& McCullagh, P. (2004). Cultural Influence on Youth's Motivation of Participation in Physical Activity. Journal of sport Behavior, 27(4).

Yang, W. (2013). The problems and solutions of the school transitions in China. Studies in Early Childhood Education, 8, 61-63. 


\section{Appendix:}

The sampling plan defined a cell structure that identified the appropriate number of children for each cell. The cells were defined in terms of 7 levels of geographic region, 2 levels of gender, 10 levels of age, and 4 levels of parental educational level. The plan was to recruit 2000 children which could be best representative to the national population, and a total of 2185 children from 51 nurseries and schools over the country were recruited for the study.

The 2010 Census divides China into 7 geographic regions: Northeast China, North China, East China, South China, Southwest China, Northwest China, and Central China. The number of children required for each cell of the sample is in accordance with the proportions of the China population between age 3:0-10:11 years living in each region according to the 2010 Census. Table A1 shows the breakdown of number of children targeted and actually recruited in each geographic region.

\section{(insert Table A1 here)}

The education level of the parents was used to indicate SES. Information on educational level was obtained from parental consent forms. Effort was made to ensure that the proportion of children of parents from each educational level would be proportionate to the distribution of these educational levels in the equivalent age group within the population at large (Table A2).

\section{(insert Table A2 here)}


Table 1 The number of children recruited in each age and gender group

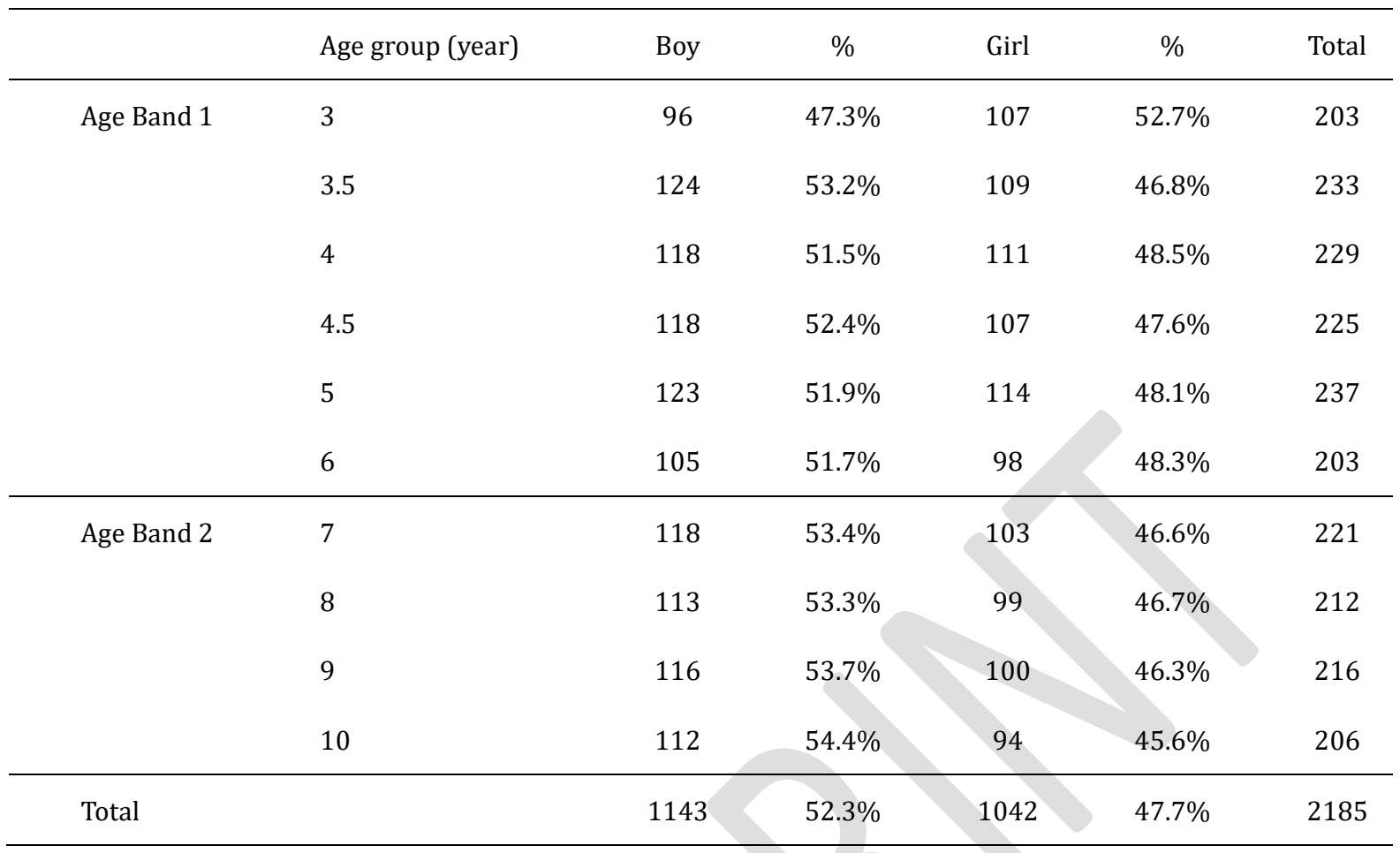


Table 2 Tasks and measurements in Movement ABC-2

\begin{tabular}{|c|c|c|c|c|c|}
\hline $\begin{array}{l}\text { Movement ABC-2 } \\
\text { test motor domain }\end{array}$ & Task- AB1 & Task-AB2 & Accuracy & Time & Actual raw score \\
\hline \multirow{3}{*}{ Manual Dexterity } & $\begin{array}{l}\text { MD1 posting } \\
\text { coins }\end{array}$ & MD1 placing pegs & $\sqrt{ }$ & $\sqrt{ }$ & Completion time \\
\hline & $\begin{array}{l}\text { MD2 threading } \\
\text { beads }\end{array}$ & MD2 threading lace & $\sqrt{ }$ & $\sqrt{ }$ & Completion time \\
\hline & $\begin{array}{l}\text { MD3 drawing } \\
\text { trail }\end{array}$ & MD3 drawing trail & $\sqrt{ }$ & & $\begin{array}{l}\text { Number of errors } \\
\text { made }\end{array}$ \\
\hline \multirow[b]{2}{*}{ Aiming and Catching } & $\begin{array}{l}\text { AC1 catching } \\
\text { beanbag }\end{array}$ & $\begin{array}{l}\text { AC1 catching with two } \\
\text { hands }\end{array}$ & $\sqrt{ }$ & & $\begin{array}{l}\text { Number of catches } \\
\text { out of } 10\end{array}$ \\
\hline & $\begin{array}{l}\text { AC2 throwing } \\
\text { beanbag onto } \\
\text { mat }\end{array}$ & $\begin{array}{l}\text { AC2 throwing beanbag } \\
\text { onto mat }\end{array}$ & $\sqrt{ }$ & & $\begin{array}{l}\text { Number of catches } \\
\text { out of } 10\end{array}$ \\
\hline \multirow{3}{*}{ Balance } & $\begin{array}{l}\text { BAL1 one-leg } \\
\text { balance }\end{array}$ & $\begin{array}{l}\text { BAL1 one-board } \\
\text { balance }\end{array}$ & $\sqrt{ }$ & & $\begin{array}{l}\text { Time to keep balance } \\
\text { as required }\end{array}$ \\
\hline & $\begin{array}{l}\text { BAL2 walking } \\
\text { heels raised }\end{array}$ & $\begin{array}{l}\text { BAL2 walking heel-to- } \\
\text { toe forwards }\end{array}$ & $\sqrt{ }$ & & $\begin{array}{l}\text { Number of steps } \\
\text { made as required }\end{array}$ \\
\hline & $\begin{array}{l}\text { BAL3 jumping on } \\
\text { mats }\end{array}$ & BAL3 hopping on mats & $\sqrt{ }$ & & $\begin{array}{l}\text { Number of } \\
\text { jumps/hops made as } \\
\text { required }\end{array}$ \\
\hline
\end{tabular}


Table 3 Mean scores and standard deviation of the Chinese and UK samples in Age Band 1.

\begin{tabular}{|c|c|c|c|c|c|c|c|c|c|c|c|c|c|c|c|c|c|c|c|c|c|c|}
\hline \multirow[b]{2}{*}{ Age } & \multirow[b]{2}{*}{ Country } & \multirow[b]{2}{*}{ Gender } & \multicolumn{2}{|c|}{ MD1-pref } & \multicolumn{2}{|c|}{ MD1-npref } & \multicolumn{2}{|c|}{ MD2 } & \multicolumn{2}{|c|}{ MD3 } & \multicolumn{2}{|c|}{ AC1 } & \multicolumn{2}{|c|}{$\mathrm{AC} 2$} & \multicolumn{2}{|c|}{ BAL1-best } & \multicolumn{2}{|c|}{ BAL1-other } & \multicolumn{2}{|c|}{ BAL2 } & \multicolumn{2}{|c|}{ BAL3 } \\
\hline & & & Mean & $\mathrm{SD}$ & Mean & $\mathrm{SD}$ & Mean & SD & Mean & SD & Mean & SD & Mean & SD & Mean & SD & Mean & SD & Mean & SD & Mean & SD \\
\hline \multirow[t]{4}{*}{3} & China & Male & 13.79 & 3.70 & 17.04 & 7.90 & 62.18 & 25.58 & 8.43 & 6.81 & 4.30 & 2.65 & 2.41 & 1.87 & 3.69 & 2.99 & 1.92 & 1.84 & 5.97 & 5.27 & 3.85 & 1.72 \\
\hline & & Female & 13.96 & 4.29 & 16.80 & 6.88 & 51.64 & 16.77 & 7.28 & 6.82 & 4.35 & 2.83 & 2.04 & 2.01 & 4.94 & 4.50 & 2.68 & 2.61 & 6.61 & 5.24 & 4.19 & 1.43 \\
\hline & UK & Male & 14.41 & 2.75 & 16.59 & 4.94 & 60.37 & 21.75 & 7.96 & 4.81 & 4.22 & 2.17 & 2.93 & 1.80 & 4.89 & 4.41 & 2.78 & 2.39 & 6.22 & 3.78 & 3.07 & 1.52 \\
\hline & & Female & 13.39 & 2.35 & 15.78 & 5.34 & 54.22 & 15.40 & 5.52 & 2.54 & 4.48 & 2.00 & 3.17 & 1.90 & 5.87 & 5.79 & 3.52 & 3.06 & 7.17 & 3.24 & 3.70 & 1.06 \\
\hline \multirow[t]{4}{*}{3.5} & China & Male & 11.28 & 2.75 & 13.92 & 5.26 & 50.15 & 26.54 & 4.52 & 4.48 & 5.66 & 2.61 & 3.59 & 2.29 & 8.12 & 6.91 & 4.34 & 3.85 & 8.35 & 5.42 & 4.49 & 1.13 \\
\hline & & Female & 11.72 & 2.55 & 13.72 & 4.26 & 42.62 & 19.81 & 3.61 & 4.30 & 5.40 & 2.45 & 3.10 & 2.32 & 11.04 & 8.67 & 5.75 & 5.44 & 10.22 & 5.16 & 4.68 & 0.83 \\
\hline & UK & Male & 12.58 & 3.27 & 14.12 & 3.00 & 55.91 & 21.63 & 6.76 & 5.66 & 5.88 & 2.42 & 3.21 & 2.04 & 4.94 & 4.70 & 2.91 & 2.70 & 9.42 & 4.90 & 3.94 & 1.25 \\
\hline & & Female & 11.64 & 2.46 & 14.33 & 4.80 & 42.06 & 15.09 & 5.52 & 4.56 & 6.09 & 1.74 & 3.52 & 2.21 & 7.67 & 7.23 & 4.45 & 4.37 & 10.42 & 5.07 & 4.58 & 0.90 \\
\hline \multirow[t]{4}{*}{4} & China & Male & 10.52 & 2.62 & 11.52 & 2.63 & 36.47 & 16.51 & 2.92 & 2.96 & 6.79 & 2.11 & 4.24 & 2.17 & 11.86 & 8.55 & 6.22 & 5.36 & 9.27 & 5.34 & 4.81 & 0.57 \\
\hline & & Female & 10.62 & 3.61 & 12.79 & 6.40 & 37.20 & 19.33 & 1.66 & 2.07 & 6.32 & 2.29 & 3.91 & 1.99 & 14.26 & 9.80 & 8.88 & 8.05 & 9.96 & 5.31 & 4.89 & 0.43 \\
\hline & UK & Male & 10.66 & 2.29 & 12.46 & 3.68 & 39.85 & 15.04 & 4.80 & 4.69 & 6.51 & 2.57 & 4.51 & 1.99 & 8.73 & 6.01 & 5.20 & 4.64 & 10.27 & 5.02 & 4.56 & 0.92 \\
\hline & & Female & 10.79 & 2.77 & 12.67 & 3.73 & 35.55 & 16.90 & 3.67 & 4.55 & 7.03 & 2.26 & 3.91 & 2.08 & 12.00 & 9.56 & 8.36 & 7.77 & 11.52 & 4.34 & 4.61 & 0.93 \\
\hline \multirow[t]{4}{*}{4.5} & China & Male & 10.08 & 2.74 & 10.91 & 3.03 & 35.75 & 13.50 & 1.98 & 2.23 & 7.01 & 2.10 & 4.61 & 2.08 & 15.89 & 9.05 & 9.09 & 6.90 & 10.34 & 4.84 & 4.96 & 0.20 \\
\hline & & Female & 9.93 & 2.90 & 11.29 & 2.92 & 32.12 & 11.48 & 1.80 & 2.68 & 7.10 & 2.43 & 4.81 & 2.33 & 20.23 & 10.00 & 13.21 & 9.79 & 12.47 & 4.06 & 4.95 & 0.29 \\
\hline & UK & Male & 9.97 & 1.80 & 11.17 & 2.52 & 35.94 & 18.26 & 2.03 & 1.98 & 7.53 & 1.86 & 5.47 & 1.70 & 14.72 & 9.05 & 10.50 & 7.88 & 11.33 & 3.99 & 4.64 & 0.49 \\
\hline & & Female & 10.49 & 3.16 & 11.71 & 3.85 & 28.60 & 8.25 & 1.49 & 1.56 & 7.49 & 1.72 & 4.71 & 2.26 & 18.11 & 8.73 & 11.03 & 7.31 & 11.89 & 3.43 & 4.60 & 0.81 \\
\hline \multirow[t]{4}{*}{5} & China & Male & 17.82 & 2.34 & 20.26 & 2.91 & 54.93 & 14.76 & 0.98 & 1.25 & 7.30 & 2.10 & 6.33 & 2.02 & 22.79 & 8.73 & 16.49 & 9.91 & 12.54 & 4.41 & 4.90 & 0.35 \\
\hline & & Female & 17.34 & 2.92 & 19.36 & 3.12 & 47.46 & 13.64 & 0.82 & 1.27 & 7.20 & 2.08 & 5.60 & 1.93 & 24.43 & 7.72 & 19.22 & 10.04 & 13.40 & 3.33 & 4.91 & 0.39 \\
\hline & UK & Male & 18.90 & 3.20 & 21.44 & 4.25 & 54.33 & 21.09 & 1.13 & 1.42 & 6.92 & 2.47 & 6.06 & 2.20 & 18.23 & 10.13 & 12.44 & 8.91 & 13.31 & 3.16 & 4.52 & 0.95 \\
\hline & & Female & 18.89 & 3.43 & 21.54 & 4.02 & 48.04 & 11.89 & 0.33 & 1.01 & 6.48 & 2.93 & 5.22 & 1.81 & 20.41 & 10.09 & 14.17 & 9.14 & 13.17 & 3.69 & 4.57 & 1.00 \\
\hline \multirow[t]{4}{*}{6} & China & Male & 16.13 & 2.05 & 17.96 & 2.62 & 44.61 & 10.82 & 0.43 & 0.76 & 8.36 & 1.42 & 6.87 & 1.96 & 26.85 & 6.01 & 21.90 & 9.33 & 14.02 & 2.71 & 4.90 & 0.46 \\
\hline & & Female & 15.96 & 2.29 & 17.97 & 2.69 & 41.21 & 11.50 & 0.44 & 1.01 & 8.32 & 1.61 & 6.69 & 1.91 & 28.60 & 4.55 & 25.30 & 7.73 & 14.74 & 1.58 & 4.94 & 0.37 \\
\hline & UK & Male & 18.02 & 3.02 & 19.62 & 2.99 & 46.51 & 9.96 & 0.57 & 0.71 & 8.51 & 1.94 & 6.30 & 2.00 & 20.87 & 10.00 & 15.89 & 10.97 & 13.30 & 3.54 & 4.55 & 0.97 \\
\hline & & Female & 17.57 & 2.62 & 19.23 & 3.22 & 40.63 & 10.55 & 0.07 & 0.37 & 8.07 & 2.29 & 6.20 & 2.12 & 26.37 & 6.40 & 21.03 & 9.63 & 14.73 & 1.46 & 4.83 & 0.46 \\
\hline
\end{tabular}


Table 4 Mean scores and standard deviation of the Chinese and UK samples in Age Band 2

\begin{tabular}{|c|c|c|c|c|c|c|c|c|c|c|c|c|c|c|c|c|c|c|c|c|c|c|c|c|}
\hline \multirow[b]{2}{*}{ Age } & \multirow[b]{2}{*}{ Country } & \multirow[b]{2}{*}{ Gender } & \multicolumn{2}{|c|}{ MD1-pref } & \multicolumn{2}{|c|}{ MD1-npref } & \multicolumn{2}{|c|}{ MD2 } & \multicolumn{2}{|c|}{ MD3 } & \multicolumn{2}{|c|}{$\mathrm{AC} 1$} & \multicolumn{2}{|c|}{ AC2 } & \multicolumn{2}{|c|}{ BAL1-best } & \multicolumn{2}{|c|}{ BAL1-other } & \multicolumn{2}{|c|}{ BAL2 } & \multicolumn{2}{|c|}{ BAL3-best } & \multicolumn{2}{|c|}{ BAL3-other } \\
\hline & & & Mean & SD & Mean & SD & Mean & SD & Mean & SD & Mean & SD & Mean & SD & Mean & SD & Mean & SD & Mean & SD & Mean & SD & Mean & SD \\
\hline \multirow[t]{4}{*}{7} & China & Male & 29.88 & 5.77 & 33.22 & 6.43 & 35.47 & 24.98 & 1.00 & 1.37 & 4.75 & 2.87 & 5.80 & 1.96 & 17.14 & 10.03 & 8.63 & 7.85 & 13.31 & 3.66 & 4.84 & 0.51 & 4.41 & 1.10 \\
\hline & & Female & 29.19 & 5.18 & 33.21 & 7.39 & 33.23 & 23.18 & 1.04 & 1.57 & 4.06 & 2.81 & 4.99 & 2.02 & 21.19 & 9.43 & 12.69 & 9.94 & 14.15 & 2.83 & 4.84 & 0.56 & 4.47 & 1.04 \\
\hline & UK & Male & 33.90 & 10.17 & 37.69 & 7.85 & 33.10 & 15.91 & 1.19 & 1.92 & 6.31 & 3.14 & 6.48 & 2.04 & 14.21 & 9.82 & 6.81 & 5.83 & 12.90 & 4.21 & 4.57 & 1.04 & 3.81 & 1.40 \\
\hline & & Female & 31.17 & 7.61 & 35.74 & 8.13 & 29.91 & 7.40 & 0.81 & 1.04 & 5.45 & 2.39 & 5.98 & 2.17 & 20.04 & 9.16 & 12.13 & 9.03 & 14.06 & 2.13 & 4.89 & 0.37 & 4.38 & 0.85 \\
\hline \multirow[t]{4}{*}{8} & China & Male & 27.27 & 4.18 & 30.77 & 5.20 & 28.51 & 8.44 & 0.73 & 1.01 & 5.88 & 2.96 & 6.05 & 2.30 & 20.19 & 9.74 & 12.43 & 9.57 & 13.62 & 3.21 & 4.89 & 0.43 & 4.50 & 1.09 \\
\hline & & Female & 26.01 & 4.17 & 29.55 & 4.91 & 26.85 & 9.59 & 0.48 & 0.79 & 5.23 & 2.83 & 5.40 & 2.04 & 24.18 & 8.49 & 16.88 & 10.14 & 14.80 & 1.12 & 4.96 & 0.32 & 4.58 & 1.02 \\
\hline & UK & Male & 27.63 & 4.41 & 31.74 & 6.29 & 27.37 & 7.26 & 0.74 & 1.52 & 7.45 & 2.51 & 7.47 & 1.47 & 17.47 & 9.54 & 9.97 & 8.66 & 13.53 & 3.16 & 4.74 & 0.69 & 4.45 & 1.03 \\
\hline & & Female & 26.33 & 4.57 & 31.33 & 6.58 & 26.54 & 7.47 & 0.33 & 0.78 & 7.15 & 2.44 & 6.35 & 1.64 & 21.65 & 8.93 & 13.63 & 8.72 & 14.21 & 2.41 & 4.83 & 0.52 & 4.52 & 0.99 \\
\hline \multirow[t]{4}{*}{9} & China & Male & 26.01 & 4.04 & 29.21 & 4.83 & 25.38 & 6.51 & 0.39 & 0.60 & 5.06 & 2.79 & 6.80 & 2.04 & 21.30 & 8.99 & 11.37 & 8.35 & 14.37 & 2.10 & 4.98 & 0.13 & 4.78 & 0.55 \\
\hline & & Female & 25.76 & 3.79 & 28.50 & 4.57 & 22.70 & 5.06 & 0.51 & 0.98 & 3.48 & 2.78 & 6.49 & 1.89 & 25.07 & 8.19 & 16.83 & 10.10 & 14.84 & 1.20 & 4.96 & 0.28 & 4.76 & 0.65 \\
\hline & UK & Male & 27.23 & 5.30 & 31.16 & 4.98 & 25.06 & 7.62 & 0.29 & 0.53 & 7.03 & 3.03 & 7.06 & 2.37 & 19.90 & 9.72 & 12.23 & 9.66 & 14.29 & 2.05 & 4.74 & 0.68 & 4.13 & 1.28 \\
\hline & & Female & 26.30 & 4.77 & 30.88 & 5.75 & 23.30 & 4.54 & 0.43 & 1.24 & 4.50 & 3.58 & 7.13 & 1.99 & 23.58 & 8.50 & 17.18 & 11.25 & 14.58 & 1.41 & 4.88 & 0.40 & 4.50 & 0.72 \\
\hline \multirow[t]{4}{*}{10} & China & Male & 25.49 & 4.02 & 27.95 & 4.23 & 24.71 & 8.41 & 0.55 & 0.98 & 6.71 & 2.80 & 7.34 & 1.85 & 22.85 & 9.12 & 16.07 & 10.85 & 14.38 & 2.21 & 4.97 & 0.16 & 4.85 & 0.57 \\
\hline & & Female & 24.69 & 3.71 & 27.06 & 4.06 & 20.68 & 5.33 & 0.23 & 0.58 & 4.49 & 2.99 & 7.11 & 1.90 & 26.12 & 6.99 & 19.60 & 10.26 & 14.86 & 1.01 & 4.99 & 0.10 & 4.83 & 0.56 \\
\hline & UK & Male & 25.25 & 3.10 & 28.80 & 5.38 & 24.55 & 7.22 & 0.43 & 0.95 & 8.34 & 2.18 & 8.14 & 1.66 & 24.27 & 7.82 & 16.98 & 10.86 & 14.66 & 1.43 & 4.89 & 0.49 & 4.48 & 1.02 \\
\hline & & Female & 25.23 & 3.83 & 29.37 & 6.55 & 21.30 & 5.87 & 0.51 & 1.03 & 5.63 & 3.36 & 7.23 & 1.95 & 22.28 & 9.15 & 16.21 & 10.66 & 14.70 & 1.58 & 4.95 & 0.21 & 4.56 & 0.98 \\
\hline
\end{tabular}


Table 5. Multivariate Analysis of Variance. Univariate F-tests of significance. Movement ABC-2 Age Band 1.

\begin{tabular}{lllllll}
\hline & \multicolumn{5}{c}{ Factor effect } \\
\cline { 2 - 6 } & Country & & & Sex & & Age \\
\cline { 2 - 6 } Task & $\mathrm{F}$ & Sig of F & $\mathrm{F}$ & Sig of F & $\mathrm{F}$ & Sig of F \\
\hline MD1-pref & 16.92 & $<0.001$ & 0.89 & .346 & 312.21 & $<0.001$ \\
MD1-npref & 5.77 & .016 & $<0.001$ & .946 & 165.50 & $<0.001$ \\
MD2 & 0.24 & .623 & 42.65 & $<0.001$ & 54.24 & $<0.001$ \\
MD3 & 4.35 & .037 & 18.79 & $<0.001$ & 111.23 & $<0.001$ \\
AC1 & 0.50 & .480 & 0.19 & .663 & 67.65 & $<0.001$ \\
AC2 & 0.53 & .467 & 6.77 & .009 & 101.25 & $<0.001$ \\
BAL1-best & 31.75 & $<0.001$ & 37.20 & $<0.001$ & 213.41 & $<0.001$ \\
BAL1-other & 20.79 & $<0.001$ & 31.46 & $<0.001$ & 186.49 & $<0.001$ \\
BAL2 & 2.64 & .105 & 15.94 & $<0.001$ & 73.31 & $<0.001$ \\
BAL3 & 56.14 & $<0.001$ & 15.08 & $<0.001$ & 43.69 & $<0.001$ \\
\hline
\end{tabular}


Table 6. Multivariate Analysis of Variance. Interaction effects, univariate F-tests of significance. Movement ABC-2 Age Band 1.

\begin{tabular}{lllllll}
\hline & \multicolumn{5}{c}{ Interaction } \\
\cline { 2 - 7 } Task & \multicolumn{1}{c}{ Country by Sex } & \multicolumn{2}{c}{ Country by Age } & \multicolumn{2}{l}{ Sex by Age } \\
\cline { 2 - 7 } MD1-pref & 0.72 & .397 & 3.07 & .009 & 0.36 & .876 \\
MD1-npref & 0.02 & .880 & 2.01 & .074 & 0.68 & .640 \\
MD2 & 1.07 & .301 & 0.34 & .886 & 1.70 & .132 \\
MD3 & 1.60 & .207 & 6.76 & $<0.001$ & 1.38 & .228 \\
AC1 & 0.27 & .606 & 1.71 & .130 & 0.29 & .920 \\
AC2 & 0.01 & .922 & 2.72 & .019 & 1.09 & .365 \\
BAL1-best & 0.50 & .481 & 2.99 & .011 & 0.85 & .511 \\
BAL1-other & 0.20 & .656 & 5.65 & $<0.001$ & 1.31 & .256 \\
BAL2 & 0.39 & .533 & 0.82 & .539 & 0.46 & .807 \\
BAL3 & 2.67 & .102 & 1.25 & .283 & 2.98 & .011 \\
\hline
\end{tabular}


Table 7 Multivariate Analysis of Variance. Univariate F-tests of significance for Age Band 2.

\begin{tabular}{|c|c|c|c|c|c|c|}
\hline \multirow[b]{3}{*}{ Task } & \multicolumn{6}{|c|}{ Factor effect } \\
\hline & \multicolumn{2}{|c|}{ Country } & \multicolumn{2}{|l|}{ Sex } & \multicolumn{2}{|l|}{ Age } \\
\hline & $\mathrm{F}$ & Sig of $F$ & $\mathrm{~F}$ & Sig of F & $\mathrm{F}$ & Sig of $F$ \\
\hline MD1-pref & 11.79 & .001 & 9.82 & .002 & 68.18 & $<0.001$ \\
\hline MD1-npref & 33.67 & .000 & 2.70 & .101 & 61.38 & $<0.001$ \\
\hline MD2 & 0.95 & .329 & 8.95 & .003 & 31.34 & $<0.001$ \\
\hline MD3 & 0.13 & .715 & 2.94 & .087 & 16.18 & $<0.001$ \\
\hline AC1 & 67.50 & $<0.001$ & 60.51 & $<0.001$ & 15.90 & $<0.001$ \\
\hline AC2 & 32.25 & $<0.001$ & 18.68 & $<0.001$ & 31.34 & $<0.001$ \\
\hline BAL1-best & 9.75 & .002 & 32.65 & $<0.001$ & 18.37 & $<0.001$ \\
\hline BAL1-other & 3.53 & .060 & 37.74 & $<0.001$ & 23.64 & $<0.001$ \\
\hline BAL2 & 1.21 & .272 & 16.50 & $<0.001$ & 9.38 & $<0.001$ \\
\hline BAL3-best & 17.84 & $<0.001$ & 9.23 & .002 & 6.15 & $<0.001$ \\
\hline BAL3-other & 24.15 & $<0.001$ & 6.29 & .012 & 8.73 & $<0.001$ \\
\hline
\end{tabular}


Table 8 Multivariate Analysis of Variance. Interaction effects, univariate F-tests of significance for Age Band 2.

\begin{tabular}{lllllll}
\hline & \multicolumn{5}{c}{ Interaction } \\
\cline { 2 - 7 } Task & \multicolumn{7}{c}{ Country by Sex } & \multicolumn{2}{c}{ Country by Age } & \multicolumn{2}{c}{ Sex by Age } \\
\cline { 2 - 7 } MD1-pref & $\mathrm{F}$ & Sig of F & $\mathrm{F}$ & Sig of F & F & Sig of F \\
MD1-npref & 0.60 & .438 & 4.47 & .004 & 0.93 & .424 \\
MD2 & 0.07 & .798 & 1.75 & .155 & 0.25 & .862 \\
MD3 & 0.06 & .807 & 0.79 & .501 & 0.38 & .768 \\
AC1 & 0.09 & .762 & 0.28 & .838 & 1.65 & .175 \\
AC2 & 0.74 & .391 & 0.17 & .914 & 6.89 & $<0.001$ \\
BAL1-best & 0.20 & .656 & 1.84 & .137 & 1.43 & .234 \\
BAL1-other & 0.53 & .466 & 0.30 & .827 & 2.67 & .046 \\
BAL2 & 0.76 & .384 & 1.22 & .300 & 1.87 & .133 \\
BAL3-best & 0.41 & .522 & 0.30 & .825 & 1.44 & .229 \\
BAL3-other & 6.06 & .014 & 0.61 & .611 & 0.99 & .397 \\
\hline & 4.43 & .036 & 1.96 & .118 & 1.20 & .309 \\
\hline
\end{tabular}


Table A1 The number of children targeted and actually recruited in each region

\begin{tabular}{lllll} 
& Actual sample & \multicolumn{3}{c}{$\begin{array}{c}\text { Targeted (according to } \\
\text { the 2010 Census) }\end{array}$} \\
& $\mathrm{N}$ & $\%$ & $\mathrm{~N}$ & $\%$ \\
Northeast China & 179 & $8.2 \%$ & 170 & $8.5 \%$ \\
North China & 367 & $16.8 \%$ & 258 & $12.9 \%$ \\
East China & 701 & $32.1 \%$ & 662 & $33.1 \%$ \\
South China & 335 & $15.3 \%$ & 325 & $16.3 \%$ \\
Southwest China & 197 & $9.0 \%$ & 185 & $9.2 \%$ \\
Northeast China & 141 & $6.5 \%$ & 126 & $6.3 \%$ \\
Central China & 265 & $12.1 \%$ & 274 & $13.7 \%$ \\
\hline Total & 2185 & & 2000 & \\
\hline
\end{tabular}


Table A2 Average length of education of parents ${ }^{3}$

\begin{tabular}{lllll}
\hline $\begin{array}{l}\text { Length of education } \\
\text { years of parents }\end{array}$ & Mother & & Father & \\
\hline & $\mathrm{N}$ & $\%$ & $\mathrm{~N}$ & $\%$ \\
\hline$<9$ years & 172 & 8.8 & 147 & 7.5 \\
\hline $9-12$ & 374 & 19.2 & 350 & 17.9 \\
\hline $12-16$ & 1047 & 53.6 & 1081 & 55.3 \\
\hline$>17$ years & 360 & 18.4 & 378 & 19.3 \\
\hline Total & 1953 & & 1956 & \\
\hline
\end{tabular}

3 For 229 children, information on the parents' education was missing, leaving data for 1956. The missing data was considered to be random and therefore should not affect the sample or the results. 